\title{
Health-seeking behavior of Filipino migrants in Australia: The influence of persisting acculturative stress and depression
}

\section{Della Maneze, MD (Corresponding Author)}

Multicultural Health Promotion Officer

South Western Sydney Local Health District

59a Cumberland Rd., Ingleburn, NSW, Australia 2565

University of Technology Sydney, Australia

Faculty of Health

PO Box 123, Broadway NSW, Australia 2007

T: 612 87884200|F: 612 96182219|E: della.maneze@sswahs.nsw.gov.au

\section{Yenna Salamonson RN PhD}

Associate Professor

University of Western Sydney

School of Nursing and Midwifery

Centre for Applied Nursing Research

Affiliate Member, Ingham Institute for Applied Medical Research

Locked Bag 1797, Penrith, NSW, Australia 2751

T: 612 46203322|F: 612 46203161|E: y.salamonson@uws.edu.au

\section{Chandra Poudel, RN}

School of Nursing and Midwifery, University of Western Sydney

Locked Bag 1797 Penrith NSW, Australia 2751

Telephone number: +612 46203322 Fax number: +612 46203161 |

Email: c.poudel@uws.edu.au

\section{Michelle DiGiacomo, PhD}

Senior Research Fellow, Cardiovascular and Chronic Care

Faculty Health

University of Technology Sydney

PO Box 123, Broadway NSW, Australia 2007

Email: michelle.digiacomo@uts.edu.au

T: 61295144818

\section{Bronwyn Everett, RN, PhD}

Associate Professor | Deputy Director, Centre for Applied Nursing Research University of Western Sydney

School of Nursing and Midwifery

Affiliate Member, Ingham Institute for Applied Medical Research

Locked Bag 1797, Penrith NSW 2751

T: 61287389360 | F + 61287389206 |E: b.everett@uws.edu.au

Patricia M. Davidson, RN, BA, ITC, MEd, PhD

Dean and Professor,John Hopkins University, School of Nursing

Professor of Cardiovascular and Chronic Care

University of Technology Sydney

Faculty of Health

PO Box 123, Broadway NSW, Australia 2007

Email: pdavidson@jhu.edu 
Health-seeking behavior of Filipino migrants in Australia: The influence of persisting acculturative stress and depression

\begin{abstract}
Background: This study examined the relationships among the constructs of acculturative stress, depression, English language usage, health literacy, and social support and the influence of these factors on health-seeking behaviors of Filipino Australians.
\end{abstract}

Methods: Using a self-administered questionnaire, 552 respondents were recruited from November 2010 to June 2011. Structural equation modelling was used to examine relationships.

Results: A direct and negative relationship between health-seeking behavior and depression, and an indirect relationship with acculturative stress, was observed mediated through depression. Social support had an important moderating influence on these effects. Although there was an inverse relationship between age and English language usage and depression, age was positively related to health-seeking behavior.

Discussion: Despite the long duration of stay, Filipino Australian migrants continued to experience acculturative stress and depression leading to lower health-seeking behavior. This study highlights the importance of screening for acculturative stress and depression in migrants and fostering social support.

Keywords: Acculturation, Health-seeking behavior, Filipino, social support, culture 


\section{Introduction}

Although immigrants are reported to be healthier than the host population upon arrival (the healthy migrant effect), their health status has been reported to approximate that of the host population over time [1]. Several reasons have been postulated for this including a change in health-seeking behavior (HSB) as they assimilate with and "fit" into the lifestyle of the destination culture resulting in an increasing similarity in health outcomes with the native population [2]. Further, the stresses associated with settling into a new country in the process of acculturation have been linked with depression [3], which has a negative impact on the health and well-being of migrants.

\section{Health-seeking behavior}

While HSB can be defined narrowly as actions undertaken to restore well-being when ill (help-seeking) this study adopted a broader definition of HSB, to include wider affirmative health-promoting practices that uphold general health regardless of current health status [4]. Seeking assistance for health care and maintaining health are dynamic processes that evolve with the changing phases of the acculturative adaptation of migrants, through interactive experiences in the host culture [5]. The study of HSB is important as it relates to the priority and emphasis migrants place on maintaining health in the adoptive country. For the host country, greater awareness of the HSB of migrants has socioeconomic benefits and implications in the formulation of health policies that are responsive to their needs.

In addition to inherent cultural beliefs about health and illness [6], a number of host factors affect HSB. The unfamiliar sociocultural and political milieu, language barriers, socioeconomic difficulties, unfamiliar environmental conditions, different health systems and policies are some of the factors that add to consequent changes in HSB [7]. Among Filipino immigrants, it has been reported that acculturation to the Western culture (example to the 
United States of America (USA) and Australia) induces a negative change in health behaviors, contributing to an increase in incidence of chronic lifestyle diseases [8].

\section{Acculturative stress and depression in Filipinos}

Much has been written about acculturative stress and depression, but little is known about the effects on HSB in Filipino immigrants to Australia. Migration from the Philippines to English-speaking Western countries like the USA[9] and Australia [10], has increased significantly over the past decades, largely propelled by socioeconomic factors. Filipinos can be distinguished from other immigrants by their high self-rated English language skills [11], and familiarity with the Western culture as a consequence of the long history of American colonization, which are perceived to be facilitators of acculturation. Despite these advantages, Filipino migrants experience acculturative stress from socioeconomic difficulties, social isolation and cultural maladjustments, analogous to other migrant groups [12]. Consequently, studies in Western countries reported similar or even higher rate of depression compared with Caucasians and other Asian immigrant populations [13], with a disproportionate low utilization of mental health services attributed to cultural and language barriers [14]. Furthermore, physical health is also affected, with increasing obesity, diabetes and hypertension in this community, partly due to lower physical activity, changes in dietary patterns and increased uptake of unhealthy habits such as smoking with increasing duration of settlement [15].

In this study, we examined a number of factors reported to influence HSB among migrants namely: age, proficiency in the host language, health literacy, social support and religiosity and the inter-relationships of these factors to HSB, depression and acculturative stress. Social support and religiosity have been well-documented to buffer the effects of acculturative stress, reduce depression and alleviate social isolation[16]. In addition, host language 
proficiency and health literacy are known to influence health among migrants[2]. This study explored their relationships to religiosity, social support, acculturative stress, depression and HSB.

Social ecological theory [17], which guides this study design and interpretation of findings, postulates that human behavior is shaped by the dynamic interaction of environmental and personal factors which affects behavior. An example could be as simple as a change in climate to the more complex discriminatory host attitudes and practices that may contribute to the experience of acculturative stress and negative psychological well-being of migrants [18]. In contrast, personal resources such as English language skills and social network have been reported to have positive influences on the acculturative processes [19]. The main focus of this paper is to examine the inter-relationships of these factors on HSB among Filipino immigrants in Australia. The study proposes direct and indirect relationships between age, social support, depression on HSB, and indirect relationships between English language usage, acculturative stress, health literacy and religiosity on HSB (Figure1).

\section{Methods}

Participants were recruited from November 2010 to June 2011 through snowballing method, in Filipino specific community, using religious-based events, emails, advertisements in Filipino GP practices and in Filipino specific web, broadcast, print and social media like (www.facebook.com) as channels for recruitment. All participants who responded were included if they were of Filipino heritage, aged 18 years and older and currently living in Australia. Those who participated were given the choice of answering the questionnaire online or given a hardcopy questionnaire. Ethics approval was obtained from two university human research ethics committees (H8617; HREC2013000645) and from the Area Health Service (HREC/13/LPOOL29). 


\section{Instruments}

In addition to the demographic data, seven validated standardized scales were included in the questionnaire to assess depression, acculturative stress, HSB, English language usage, health literacy, social support and religiosity.

\section{Depression}

Depression was assessed using the 2-item Patient Health Questionnaire (PHQ-2), derived from the first two questions of the PHQ-9, and was previously shown to be sensitive in detecting and monitoring depression [20]. Cronbach's alpha of the PHQ-2 in this study was 0.80.

\section{Acculturative stress}

The study modified the 16-item acculturative stress scale used by Nho [21] among Korean immigrants which he originally drew from the 26-item Social, Attitudinal, Familial and Environmental (SAFE) developed by Mena, Padilla and Maldonado and used by Hovey et.al.[22], to measure acculturative stress among Latino adolescents in the USA. Cronbach's alpha of this 17 -item scale in this study was 0.87 .

Health-seeking behavior (HSB)

The 14-item health behavior questionnaire modified from the study of Bausell and Bausell [23] included health monitoring, smoking and drinking and other behaviors (weight maintenance, exercise, sleep). We excluded items from Bausell's scale that did not directly relate to personal health behaviors (example: driving and smoke alarms). The scale assessed the frequency of health behavior practices with higher scores indicating higher frequencies of positive behavioral practices. In this study, the Cronbach's alpha of this 13-item scale was 0.82 . 


\section{English Language Usage Scale (ELUS-6)}

The level of language acculturation was measured using the modified 5-item English Language Acculturation Scale (ELAS) used by Salamonson et.al to measure acculturation among overseas-born nursing students [24]. In this study, we included language preference to reflect the language environment to which the participants are exposed which is reported to be important in the degree of immersion to the host culture [25]. The modified English Language Usage Scale (ELUS-6) was similar to the validated ELAS and ranged from only English to only Filipino language. Cronbach's alpha of this 6-item scale in this study was 0.89 .

\section{Health Literacy Scale}

Because of its brevity, the 3-item health literacy scale by Chew et.al.[26], was used to measure health literacy. The 3-item test included frequency of difficulty in understanding medical information, confidence in completing forms and number of times help was requested in reading hospital materials. Cronbach's alpha of this scale in this study was 0.62 .

\section{Social Support}

The study used the Duke Social Support Index (DSSI) [27], a 10-item scale, which assessed the level of social support in two dimensions: social interactions and satisfaction with the level of social support. To take into consideration contemporary social interaction modality that is also highly relevant in this community group, we added an item to include cybersocialization such as in social networking sites (Facebook, Twitter, etc.). The Cronbach's alpha of the 11-item scale in this study was 0.74 .

\section{Religiosity}


Religiosity is recognized as an important sociocultural factor of particular importance among Filipinos [28]. This study used the intrinsic religiosity subscale of the Duke University Religion Index (DUREL) [29], and the Cronbach's alpha of the three items in this study was 0.83 .

\section{Statistical analysis}

Data were collected using paper- and web-based questionnaires, manually entered and downloaded into Statistical Package for Social Science (IBM ${ }^{\circledR}$ SPSS $^{\circledR}$ ) version 21.

Descriptive statistics were used to summarize the sample characteristics using percentage, mean, range and standard deviation [30]. Chi-square or independent sample $t$-test was used to assess group differences, and bivariate correlations among variables were assessed using Pearson Product-Moment Correlation. Cronbach’s alpha was calculated to assess the reliability of each standard instrument

A hypothesized model (Figure 1) was developed to test the direct and indirect relationships of variables as well as goodness-of-fit of the model, using structural equation modeling (SEM) with AMOS version 20 [31]. We included age, English language usage, health literacy, religiosity, social support and acculturative stress as observed variables and examined the relationship to depression and HSB. The model fit was evaluated using the following indices and their cut-off values: Chi-square test ( $p$ value $>0.05$ ), comparative fit index (CFI) near 0.95 or greater, Tucker-Lewis Fit Index (TLI) near 0.90 or greater, Normed Fit Index (NFI) near 0.95 or greater and root-mean-square error of approximation (RMSEA) close to 0.06 or less for a good fitted model [30, 31].

\section{Results:}

A total of 552 participants were recruited to the study. The demographic characteristics of the study population were consistent with those reported in the 2011 ABS census [11] (Table 1). 
Most of the participants had a duration of stay in Australia of 20 years or more $(n=233$ (41.2\%) and only 63 (14.1\%) arrived five years or less.

The correlation analysis showed that significant relationships exist at $p<0.01$ among variables under study. We found the strongest negative correlation between age and acculturation level $(r=-0.43, p<.001)$ indicating that those who were older used less English and assessed as having lower acculturation level. There was a strong positive correlation between acculturative stress and depression $(r=0.30, p<0.001)$ which in turn had a significant negative and direct correlation with HSB ( $r=-0.28, p<0.001)$. Among Filipino migrants, social support had significant and direct correlation with four variables, namely, health literacy $(r=0.23$, $p<0.001)$, acculturative stress $(r=-0.32, p<0.001)$, depression $(r=-0.34, p<0.001)$ and HSB $(r=0.19, p<0.001)$. The maximum correlation coefficient of -0.43 indicated an absence of multicollinearity [30]. Table 2 illustrated the correlations among variables.

\section{Structural equation modeling (SEM) analysis}

The study used SEM to investigate the parameter estimates and model fit of our proposed model as shown in Figure 1, in order to determine the predictors of HSB among Filipino migrants. The hypothesized model fitness was found to be unsatisfactory $\left(\chi^{2}=31.57, p<0.01\right.$, TLI=0.87, CFI=0.98, NFI=0.96, RMSEA $=0.051$ ) which was then adjusted based on modification indices and theoretical assumptions. Because the result did not show a significant correlation between religiosity and acculturative stress $(r=-0.04, p>0.05)$ and depression ( $r=-0.05, p>0.05$ ), the variable religiosity was removed from further analysis to improve the parsimony of the revised model which was then tested (Figure 2).

The revised model (Figure 2) resulted in excellent fit statistics $\left(\chi^{2}=16.84, p>0.05\right.$, TLI $=0.94$, $\mathrm{CFI}=0.98, \mathrm{NFI}=0.96$, and RMSEA=0.04). Higher HSB was practiced by older people, those with higher social support and those with lower depression scores. The effect of age on 
acculturative stress was mediated indirectly through lower English language usage which in turn lowered health literacy. Higher social support was related to higher health literacy, lower acculturative stress, lower depression and higher HSB. Interestingly, there was an inverse relationship between age and depression (Figure 2).

\section{Discussion}

The result of our study showed that among our sample of Filipino migrants to Australia, depression was an important mediator of acculturative stress and HSB. The direct and positive correlation between acculturative stress and depression, and the negative effect on HSB, corroborated with previous findings that depressed people were more likely to smoke, not exercise and have an unhealthy diet [13]. Several sources of acculturative stress had been reported among Filipino immigrants [12], which could lead to higher susceptibility to depression resulting in lower HSB.

The effects of depression and acculturative stress were mitigated by higher social support. Given the importance of family and social support network in the Filipino's collectivistic cultural orientation [14, 32], it was not unexpected that social support was the only factor in our model that had direct negative effects on acculturative stress and depression and direct positive effects on HSB and health literacy underscoring the importance of this construct. This confirms findings that the network of family and friends among Filipino migrants were often the immediate source of support for emotional and mental health issues [32].

Our findings showed that age had a strong direct correlation with HSB. The effect of age is largely driven by the need for health services with aging and thus exerts important influences on HSB. The heightened awareness of physical vulnerabilities among the elderly could have accounted for the higher HSB, confirming that those who were older were more likely to assume responsibility for their health [33.] Although unexpected, the negative association 
between age and depression was similar to previously reported findings on Filipino elderly which had been attributed to lesser pre-migration trauma and higher host language proficiency compared with other Asian elderly immigrant populations [32]. However, this was refuted by other researchers suggesting that depression among Filipinos could be stigmatized, unrecognized, and somatized, and therefore likely to be underreported [14]. The results of this study could also have been due to sampling bias as most of the elderly were recruited from Filipino specific senior organizations in the area receiving strong social support and many of them were living with family members. Religiosity, while a distinctive trait among Filipinos [11] did not predict HSB in this model. The intrinsic religiosity component of the DUREL scale used in this study might not be reflective of overall religiosity of this population group.

As there is no specific Filipino word that accurately encapsulates the concept of depression, more research is needed to explore the understanding of depression in this community and the cultural context in communicating mental and emotional issues. For those who are older and are less proficient in English, providing health information with the support of the social network or through culturally congruent health workers may be beneficial in improving understanding of health issues.

It is important to note that the average duration of stay in Australia of the study participants was 20 years. The persistence of acculturative stress in this population, despite the long duration of stay, is highly significant and warrants further investigation as this directly affects the development of depression leading to negative consequences on health-seeking behaviors.

\section{Limitations of the study}

Despite being one of the top ten migrant groups to Australia in recent years [10], the lack of sampling frame for this target group in this study was a limitation[34]. Because of this, we 
chose web-based recruitment of participants, and snowballing technique, which provided cost-effective convenience enabling the research to engage a higher number of participants than would have been possible had the study been limited solely to hardcopy questionnaires. The limited participation of newly arrived Filipino migrants to Australia may have skewed the results however, the study added to the knowledge regarding factors affecting healthseeking behavior of long term migrants. The instruments used were not validated specifically for the Filipino migrant population but the internal consistency values as well as the high rates of completion suggest acceptability.

\section{Conclusion}

Among Filipino Australian immigrants, depression which is directly and positively influenced by acculturative stress had a significant negative effect on HSB. This was mitigated by having higher social support which directly and positively influence HSB. Although older Filipino migrants were more likely to engage in HSB, the limited English usage among this age group was likely to have a direct negative impact on health literacy.

Filipino migrants may have high self-rated English language proficiency and possess characteristics that may add to the perception of facilitated cultural transition and settlement. However, this study shows that despite long duration of stay in the host country, Filipino migrants continue to experience acculturative stress which may lead to depression mediating the negative consequences on health-seeking behavior. Considering the dynamic interaction of environmental and personal factors is important in formulating health care interventions. 
Table 1. Demographic characteristics of study population

\section{Characteristics}

Age, mean (SD) years, (Range: 18 - 91)

$44(13.74 \wedge)$

Sex: Female, $n(\%)$

$316\left(67^{*}\right)$

Country of birth: Philippines, $n$ (\%)

$445\left(95^{*}\right)$

Duration of stay in Australia, median ( $S D$ ) years, (Range: 0-42)

$20(9 *)$

Educational attainment: Tertiary level or higher, $n$ (\%)

$363\left(77^{*}\right)$

Language spoken at home: speaks both Filipino and English, $n$ (\%)

$383\left(81^{*}\right)$

In paid employment: Yes, $n(\%)$

$365\left(78^{*}\right)$

${ }^{\wedge}$ range * percentage 
Table 2. Bivariate correlations of variables

\begin{tabular}{|c|c|c|c|c|c|c|c|c|c|c|c|}
\hline Description & Mean & SD & $\alpha$ & Age & AS & ELUAS & REL & SS & PHQ & HSB & $\mathrm{HL}$ \\
\hline Age & 44.90 & 13.73 & & 1 & & & & & & & \\
\hline AS & 34.36 & 7.18 & 0.87 & .00 & 1 & & & & & & \\
\hline ELUAS & 21.03 & 4.75 & 0.89 & $-0.43^{* *}$ & $-0.24^{* *}$ & 1 & & & & & \\
\hline REL & 12.30 & 3.15 & 0.83 & $0.17^{* *}$ & -0.01 & $-0.19^{* *}$ & 1 & & & & \\
\hline SS & 27.56 & 3.77 & 0.74 & 0.04 & $-0.32^{* *}$ & 0.07 & 0.08 & 1 & & & \\
\hline PHQ & 2.97 & 1.40 & 0.80 & $-0.25^{* *}$ & $0.30^{* *}$ & $0.14^{* *}$ & $-0.12^{*}$ & $-0.34^{* *}$ & 1 & & \\
\hline HSB & 48.40 & 7.54 & 0.81 & $0.37^{* *}$ & -0.06 & $-0.20^{* *}$ & $0.18^{* *}$ & $0.19^{* *}$ & $-0.28^{* *}$ & 1 & \\
\hline HL & 13.21 & 2.30 & 0.62 & -0.01 & $-0.28^{* *}$ & $0.18^{* *}$ & -0.02 & $0.23^{* *}$ & $-0.12^{*}$ & 0.05 & 1 \\
\hline
\end{tabular}

Note: *. Correlation is significant at the 0.05 level (2-tailed),**. Correlation is significant at the 0.01 level (2-tailed). AS= Acculturative Stress Scale, ELUAS= English Language Usage Acculturation Scale, REL= Duke University Religion Index, SS= Duke Social Support Index, $\mathrm{PHQ}=\mathrm{Patient}$ Health Questionnaire-2, HSB=Health-Seeking Behaviour, HL=Health Literacy 


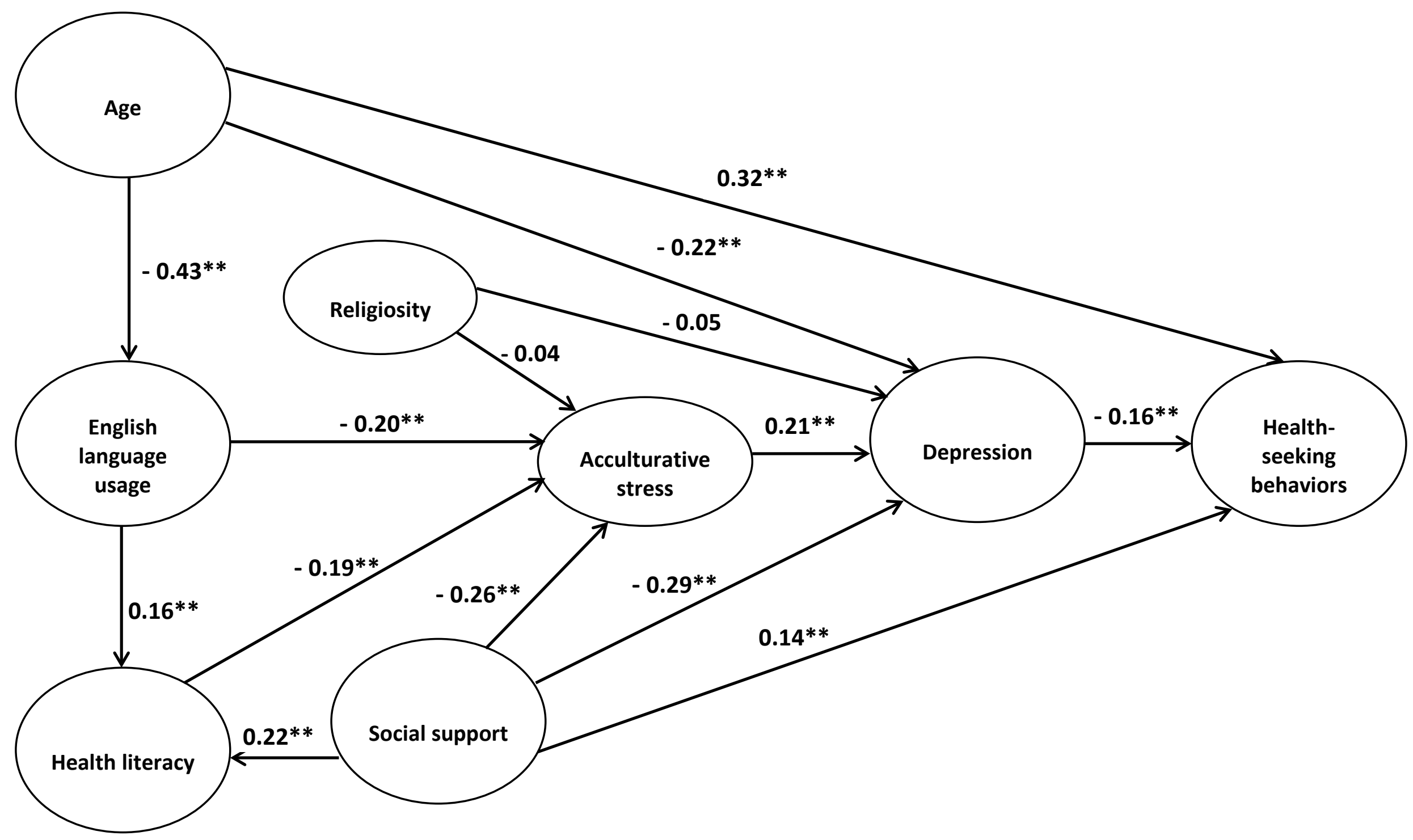

Note. Numbers provided are standardized regression weights, $n=552, \chi^{2}=31.57, \mathrm{CFI}=0.98, \mathrm{TLI}=0.87, \mathrm{NFI}=0.96 \mathrm{RMSEA}=0.05 . * * P<.01, * P<.05$

Figure 1-Hypothesized structural model: Inter-relationships of acculturative stress, depression and health-seeking behaviors among Filipino immigrants 


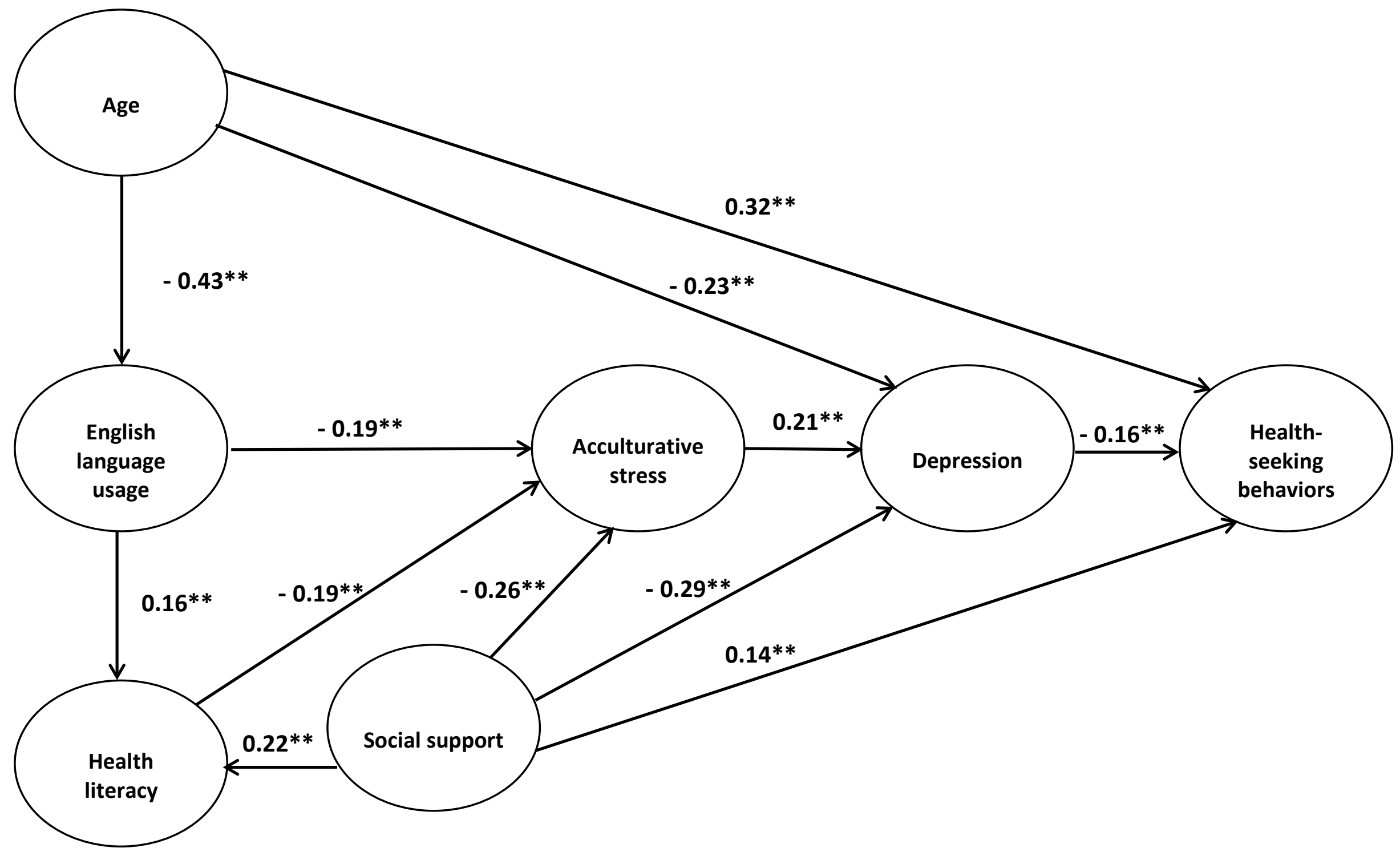

Note. Numbers provided are standardized regression weights, $n=552, \chi^{2}=16.84, \mathrm{CFI}=0.98, \mathrm{TLI}=0.94, \mathrm{NFI}=0.96 \mathrm{RMSEA}=0.04 . * * P<.01, * P<.05$

Figure 2 - Final structural model: Inter-relationships of acculturative stress, depression and health-seeking behaviors among Filipino immigrants 


\section{References}

1 Lassetter $\mathrm{JH}$, Callister LC. The impact of migration on the health of voluntary migrants in Western societies: A review of the literature. J Transcult Nurs. 2009;20:93-104.

2 Cunningham S, Ruben JD, Venkat Narayan K. Health of foreign-born people in the United States: A review. Health Place. 2008;14:623-35.

3 Hwang W-C, Ting JY. Disaggregating the effects of acculturation and acculturative stress on the mental health of Asian Americans. Cultur Divers Ethnic Minor Psychol. 2008;14:147.

Mackian S. A review of health seeking behaviour: problems and prospects. Internal concept paper Health Systems Development Programme, London School of Hygiene and Tropical Medicine London. 2001.

Choi JY. Reconstruction of health-seeking behaviors A comparative study of three Asian Pacific immigrant groups. Qual Health Res. 2013;23:517-30.

$6 \quad$ Kwok C, Sullivan G. Health seeking behaviours among Chinese-Australian women: Implications for health promotion programmes. Health (N Y). 2007;11:401-15.

7 Lorant V, Van Oyen H, Thomas I. Contextual factors and immigrants' health status: Double jeopardy. Health Place. 2008;14:678-92.

8 Serafica R. Concept analysis of acculturation in Filipino Immigrants within health context. Nurs Forum. 2011;46:128-36.

David E. A colonial mentality model of depression for Filipino Americans. Cultur Divers Ethnic Minor Psychol. 2008;14:118.

Australian Bureau of Statistics. 2004-2005 migration - Australia (3412.0). Canberra: ABS, Commonwealth of Australia; 2007.

ABS Census. 2011 QuickStats Country of Birth (Philippines). In: 2011 ABS, ed. Canberra; 2011.

Maneze D, Salamonson Y, Attwood N, Davidson PM. Acculturative stress in Filipino migrants with functional English: Implications for health promotion. Int J Cult Ment Health. 2013:1-13.

Sorkin DH, Nguyen $\mathrm{H}$, Ngo-Metzger Q. Assessing the mental health needs and barriers to care among a diverse sample of Asian American older adults. J Gen Intern Med. 2011;26:595-602.

Sanchez F, Gaw A. Mental health care of Filipino Americans. Psychiatr Serv. 2007;58:810-5.

Gentilucci U, Picardi A, Manfrini S, Khazrai Y, Fioriti E, Altomare M et al. Westernization of the Filipino population resident in Rome: obesity, diabetes and hypertension. Diabetes Metab Res Rev. 2008;24:364-70.

Aukst-Margetić B, Margetić B. Religiosity and health outcomes: Review of literature. Coll Antropol. 2005;29:365-71.

17 Stokols D. Translating social ecological theory into guidelines for community health promotion. Am J Health Promot. 1996;10:282-98. 
19 Lueck K, Wilson M. Acculturative stress in Asian immigrants: The impact of social and linguistic factors. Int J Intercult Rel. 2010;34:47-57.

Kroenke K, Spitzer RL, Williams JB. The Patient Health Questionnaire-2: Validity of a two-item depression screener. Med Care. 2003;41:1284-92.

Nho CR. Psychological well-being of Korean American and immigrant adolescents: Columbia University New York; 2000.

Hovey JD. Acculturative stress, depression, and suicidal ideation in Mexican immigrants. Cultur Divers Ethnic Minor Psychol. 2000;6:134.

Bausell C, Bausell R. The internal structure of health-seeking behavior. Eval Health Prof. 1987;10:460-75.

Salamonson Y, Everett B, Koch J, Andrew S, Davidson P. English language acculturation predicts academic performance in nursing students who speak English as a second language. Res Nurs Health. 2008;31:86-94.

Kang S-M. Measurement of acculturation, scale formats, and language competence: Their implications for adjustment. J Cross Cult Psychol. 2006;37:669-93.

Chew L, Bradley K, Boyko E. Brief questions to identify patients with inadequate health literacy. Health (N Y). 2004;11:12.

27 Powers JR, Goodger B, Byles JE. Assessment of the abbreviated Duke Social Support Index in a cohort of older Australian women. Australas J Ageing. 2004;23:71-6.

28 Tondo J. Popular religiosity and the transnational journey: Inscribing Filipino identity in the Santo Niño Fiesta in New Zealand. Asia Pac J Anthropol. 2010;11:219-44.

29 Koenig H, Büssing A. The Duke University Religion Index (DUREL): A five-item measure for use in epidemological studies. Religions. 2010;1:78-85.

30 Tabachnick BG. Using multivariate statistics. Boston: Boston : Pearson; 2013.

31 Byrne BM. Structural equation modeling with AMOS: Basic concepts, applications, and programming: CRC Press; 2009.

32 Mossakowski KN. Are immigrants healthier? The case of depression among Filipino Americans. Soc Psychol Q. 2007;70:290-304.

33 Becker CM, Arnold W. Health promoting behaviors of older Americans versus young and middle aged adults. Educ Gerontol. 2004;30:835-44.

34 Gill J, Johnson P. Research methods for managers. London: Sage; 2010. 